\title{
Phase Locking of 3.5-THz and 4.7-THz Quantum-Cascade Lasers Using a Schottky Diode Harmonic Mixer
}

\author{
Heiko Richter ${ }^{1}$, Nick Rothbart ${ }^{1,2}$, Martin Wienold ${ }^{1}$, Xiang L $\ddot{u ̈}^{3}$, Lutz Schrottke ${ }^{3}$, Holger T. Grahn ${ }^{3}$, \\ Divya Jayasankar ${ }^{4}$, Vladimir Drakinskiy ${ }^{4}$, Jan Stake ${ }^{4}$, Peter Sobis ${ }^{4,5}$, and Heinz-Wilhelm Hübers ${ }^{1,2}$ \\ ${ }^{1}$ Institute of Optical Sensor Systems, German Aerospace Center (DLR), 12489 Berlin, Germany \\ ${ }^{2}$ Department of Physics, Humboldt-Universität zu Berlin, 12489 Berlin, Germany \\ ${ }^{3}$ Paul-Drude-Institut für Festkörperelektronik, Leibniz-Institut im Forschungsverbund Berlin e. V., \\ 10117 Berlin, Germany \\ ${ }^{4}$ Terahertz and Millimetre Wave Laboratory, Department of Microtechnology and Nanoscience (MC2), \\ Chalmers University of Technology, 41296 Göteborg, Sweden \\ ${ }^{5}$ Omnisys Instruments AB, 42132 Vastra Frölunda, Sweden
}

\begin{abstract}
High frequency accuracy and stability are important requirements for local oscillators in terahertz heterodyne spectrometers. We report on a phase-locked loop for quantumcascade lasers (QCLs) operating at 3.5 and $4.7 \mathrm{THz}$, two very important frequencies in atmospheric science and astronomy. The QCLs are locked to the multiplied output of a frequency synthesizer using a Schottky diode harmonic mixer.
\end{abstract}

\section{INTRODUCTION}

$\mathrm{T}$ erahertz (THz) quantum-cascade lasers (QCLs) are of great importance as local oscillators (LOs) in high-resolution heterodyne spectrometers $[1,2]$. These spectrometers are commonly used for astronomical and atmospheric observations in the $\mathrm{THz}$ range $[2,3]$. The frequency resolution of such a spectrometer depends on the frequency stability of the QCL LO. Typically, the linewidth of a free-running THz QCL is on the order of $10 \mathrm{MHz}$. With special efforts regarding stabilization of temperature and driving current, it can be lowered to a few MHz. Stabilizing the frequency becomes even more challenging when the QCL is operated in a mechanical cryocooler, where vibration noise is intrinsically present.

In general, a powerful method for frequency stabilization of a radiation source is phase locking to a reference source. However, application of this method to THz QCLs faces two major problems: both, reference sources and mixers, are not readily available at $\mathrm{THz}$ frequencies. Mixers are a key part of the phase-locked loop (PLL), since they generate the difference frequency of the QCL and the reference source. We report on a PLL for QCLs operating at 3.5 and $4.7 \mathrm{THz}$, at which the hydroxyl radical, and atomic oxygen, respectively, have transitions. Both species are important in atmospheric science and astronomy, and several heterodyne spectrometers are currently in operation or under development for airborne and spaceborne missions $[2,4]$.

\section{RESULTS}

The QCLs which are frequency stabilized by the PLL are mounted in a compact, low-input-power Stirling cooler from AIM Infrarot-Module $\mathrm{GmbH}$. They are designed for frequencies of 3.5 and $4.7 \mathrm{THz}$. The active medium has been developed for continuous-wave operation, high output powers, and low electrical pump powers. Both QCLs are based on single-plasmon waveguides with Fabry-Pérot resonators. The cavities are sufficiently short so that single-mode emission is obtained over most of the driving current range. The beam of the QCL is formed with dedicated optics into an almost Gaussian profile. The free-running linewidth of the QCLs amounts to a few MHz. A simplified scheme of the QCL stabilization with PLL is shown in Fig. 1(a). The PLL is based on a Schottky diode harmonic mixer and a multiplier source, which pumps the harmonic mixer. The output frequency is tunable from about 570 to $610 \mathrm{GHz}$ with more than $3 \mathrm{~mW}$ of output power. The Schottky diode harmonic mixer generates the sixth and eighth harmonic for phase locking of a $3.5-\mathrm{THz}$ and a 4.7-THz QCL, respectively. Details of the design of the harmonic mixer are described in $[5,6]$. With this setup, phase locking was achieved for both frequencies [Fig. 1(b)].

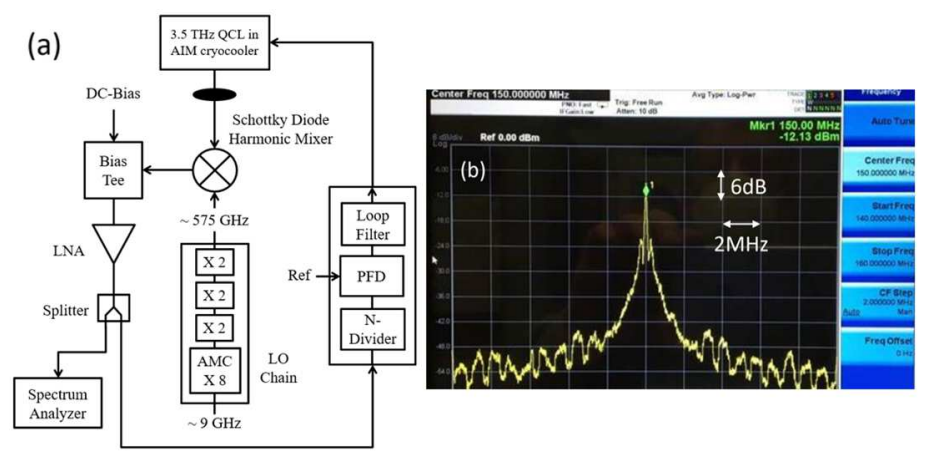

Fig. 1: (a) Scheme of the PLL setup and (b) locked signal obtained from the 3.5-THz QCL and the multiplier source.

\section{SUMMARY}

In summary, we demonstrate a PLL for THz QCLs operating at 3.5 and $4.7 \mathrm{THz}$. This paves the way for a QCL LO with a very high frequency accuracy and stability.

\section{REFERENCES}

[1] H. Richter et al., "4.7-THz local oscillator for the GREAT heterodyne spectrometer on SOFIA," IEEE Transactions on Terahertz Science and Technology, vol. 5, no. 4, pp. 539-545, 2015.

[2] C. Risacher et al., "The upGREAT dual frequency heterodyne arrays for SOFIA," Journal of Astronomical Instrumentation, vol. 7, no. 4, 1840014, 16 pages, 2018.

[3] H. Richter et al., "Direct measurements of atomic oxygen in the mesosphere and lower thermosphere using terahertz heterodyne spectroscopy", Communications Earth \& Environment, vol. 2, 19, 9 pages, 2021.

[4] S. P. Rea et al., "The low-cost upper-atmosphere sounder (LOCUS)," Proceedings of the 26th International Symposium on Space Terahertz Technology (Cambridge, MA, 2015).

[5] D. Jayasankar et al., "Development of Supra-THz Schottky diode harmonic mixers" submitted to 46th IRMMW-THz, Sep. 2021. 
[6] D. Jayasankar et al., A 3.5-THz, x6-Harmonic, Single-Ended Schottky Diode Mixer for Frequency Stabilization of Quantum-Cascade Lasers. https://doi.org/10.36227/techrxiv.14847573.v1 